\title{
Cultura popular e identidad nacional
}

\section{Popular Culture and National Identity}

\section{Cultura popular e identidade nacional}

Rafael Cuevas-Molina

Doctor en Ciencias Históricas

Académico investigador

Instituto de Estudios Latinoamericanos

Universidad Nacional, Costa Rica

Recibido: 1/02/2019

Aceptado: 15/02/2019

DOI: http://doi.org/10.15359/tdna.35-65.1

\section{Resumen}

El presente ensayo plantea consideraciones para una aproximación "des-sacralizadora" a los mitos fundantes de las nacionalidades contemporáneas, identificar los intereses de clase que se encuentran detrás de ellos, las contradicciones que esconden tras la retórica y mostrar los procesos por medio de los cuales se construyeron dichas identidades en determinadas circunstancias históricas.

Palabras clave: identidades, América Latina, Centroamérica, Estudios Culturales

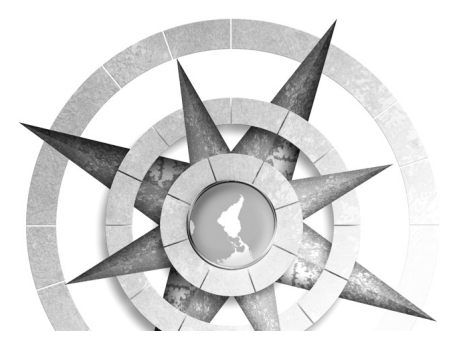

http://doi.org/10.15359/tdna.35-65.1
Abstract

The present essay raises considerations for a "de-Sacralising" approach to the founding myths of contemporary nationalities, identifying the class interests following them, the contradictions hidden behind the rhetoric and moreover, showing the processes through which those identities were constructed in given historical circumstances.

Keywords: Popular Culture, Identities, Latin America, Central America, Cultural Studies

\section{Resumo}

O presente ensaio levanta considerações para uma abordagem "da dessacralização" dos mitos fundadores das nacionalidades contemporâneas, identificando os interesses de classe por trás deles, as contradições escondidas por trás da retórica e mostrando os processos através dos quais 
essas identidades foram construídas em certas circunstâncias históricas.

Palavras chave: Culturas populares, Identidades, Latino-america, América Central, estudos culturais

Uno de los aportes más significativos de los estudios contemporáneos sobre la cultura, es la concepción según la cual las identidades no constituyen esencias inmutables, ahistóricas, siempre iguales a sí mismas, sino que, por el contrario, no son construcciones permanentes, están en constante cambio y responden a las necesidades e intereses de grupos sociales determinados. Otro aspecto importante que se ha puesto en evidencia en estos estudios a los que estamos haciendo mención, es el hecho que estos procesos de construcción de las identidades se encuentran estrechamente vinculados, firmemente entrelazados a dinámicas de tipo social, político y económico en relación con las cuales juegan el papel de legitimadores simbólicos de planes y proyectos.

En el marco de estos puntos de vista, las tradiciones que se encuentran en la base de las identidades son, en buena medida, invenciones, en el sentido que el sociólogo inglés Raymond Williams señalaba de que toda tradición es siempre una selección de algunos aspectos del pasado; selección que se hace en función de los intereses del presente que, usualmente, responden a proyectos de futuro.
Las anteriores consideraciones pueden permitir una aproximación "des-sacralizadora" a los mitos fundantes de las nacionalidades contemporáneas, identificar los intereses de clase que se encuentran detrás de ellos, las contradicciones que esconden tras los oropeles de la retórica y mostrar los procesos por medio de los cuales se construyeron en determinadas circunstancias históricas.

Si vemos hacia nuestro pasado histórico, con cierta facilidad podemos comprobar que en él existen ciertos momentos en los que esos procesos de construcción de las identidades alcanzaron especial relevancia, momentos en los cuales cristalizaron tendencias que dieron origen a perfiles identitarios de especial fuerza y permanencia en los imaginarios colectivos de nuestros países.

Uno de ellos, quizás el más importante hasta nuestros días, es el que tuvo lugar en la segunda mitad del siglo XIX, cuando en América Latina nos dimos a la tarea de construir los estados-nación modernos. En esas circunstancias históricas fue de especial importancia el problema que se planteó sobre cuál era el lugar que debía ocupar lo que hoy conocemos como culturas populares en el imaginario nacional que se constituía. En el marco del proceso modernizador impulsado por las elites gobernantes, amplios sectores de la población se resistían a incorporarse a los ritmos y a los modelos necesarios para impulsar 
las formas capitalistas de producción. $\mathrm{Su}$ resistencia fue identificada como resabios de la barbarie que los nuevos tiempos pretendían superar, y su cultura fue identificada como rezagos del tiempo pasado que debía quedar definitivamente atrás.

Pero la relación con las culturas populares no fue tan simple. Se las veía como los restos de un pasado que quería superarse a todo trance. También se las identificó como símbolo de un pasado mítico, perdido en las brumas del tiempo, en el que las congojas que imponían los ritmos de la modernidad no estaban presentes. Las culturas populares, identificadas como cultura de los sectores rurales y campesinos, fueron vistas entonces como retazos de un pasado que tenía que ver con algo así como con una edad de oro, en la que los seres humanos vivían en concordancia con la naturaleza, tenían relaciones primarias sanas, eran honrados y sabían de valores como el respeto a los mayores, la honradez, la solidaridad, etc.

La forma como los sectores dominantes se relacionaban con las culturas populares era, entonces, contradictoria. Contradictoria fue también la forma como las incorporaron a la cultura nacional que estaban en trance de construir. Un ejemplo de ello es la polémica que protagonizaron dos de las más representativas figuras de la naciente literatura costarricense, Ricardo Fernández Guardia y Carlos Gagini, en torno a cuáles eran los modelos que debían prevalecer en la literatura nacional que se estaba formando. Uno sostenía que la literatura debía ser una representación de lo real perceptible, es decir, de la realidad inmediata que rodeaba al escritor, mientras el otro argumentaba que los cánones artísticos ya habían sido definidos por la tradición cultural europea. Veamos lo que decía Fernández Guardia al respecto:

Nuestro pueblo es sandío, sin gracia alguna, desprovisto de toda poesía y originalidad que puedan dar nacimiento siquiera a una pobre sensación artística... Se comprende sin esfuerzo que con una griega de la antigüedad, dotada de esa hermosura espléndida y severa que ya no existe, se pudiera hacer una venus de Milo. De una parisiense graciosa y delicada pudo nacer la Diana de Houdon; pero vive Dios que con una india de Pacaca sólo se puede hacer otra india de Pacaca. (Quesada, 1986: 98)

También en las artes plásticas se dio esa polémica, aunque con sus propias características. Por un lado, Tomás Povedano, que entendía que el modelo a seguir era el academicista, con sus temas vinculados a lo clásico de la cultura occidental, lo cual implicaba cánones de belleza más cercanos a las estatuas griegas que alguna vez poblaran los templos de la península helénica, que la de

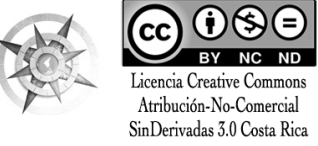


los cuerpos pequeños y morenos de los habitantes originales de estos lares, o los hirsutos y macilentos de los nuevos colonos llegados a partir del siglo XVI que poblarían estas tierras.

Esa actitud contradictoria está presente en muchos de los íconos que forman parte hoy de la cultura nacional costarricense. Como se sabe, en este período de construcción inicial se amuebló la ciudad con estatuas y monumentos que apelaban a un patriotismo cuyo referente era la joven República de Costa Rica. Uno de los acontecimientos que fueron elevados al rango de mitos fundadores de la nacionalidad fue el de la Guerra de 1856 contra los filibusteros y, en su contexto, el de la gesta del soldado Juan Santamaría. En el Parque Nacional se colocó el Monumento Nacional y en la provincia de Alajuela se instaló la estatua del joven tamborilero. Pero aunque ambas obras hacen referencia a procesos realizados por costarricenses, puede constatarse con facilidad que los rasgos de los protagonistas fundidos en el bronce, no se corresponden con los de la gente de estas latitudes. Juan Santamaría, por ejemplo, un mulato pobre de la ciudad de Alajuela, aparece con los rasgos de un garçon francés, rasgos que comparten todos los integrantes de la composición del Monumento Nacional y, también, con los de los murales del Teatro Nacional. Y estos fueron casos leves en comparación a lo sucedido, por ejemplo, en Honduras, en donde la estatua ecuestre a Francisco Morazán, que se levanta egregia en el corazón de la capital, Tegucigalpa, no es más que el Almirante Nelson, el inglés triunfador de la batalla de Trafalgar, que pareció más viril y elegante a la comisión hondureña que fue enviada a Europa a buscar un taller que fundiera la estatua del héroe que lo que podría haber sido la figura misma de Morazán.

No es de extrañar esta situación. Los grupos oligárquicos se debatían entre la contradicción de inspirarse en modelos nacionales para establecer algún tipo de vinculación de legitimidad de su proyecto político en relación con los grupos populares así como el de copiar los modelos de las culturas del otro lado del océano, a las cuales se mantenían unidos por lazos de dependencia de carácter económico. Pero aún cuando lo popular era tomado en cuenta, ese tomar en cuenta fue hecho siempre desde la perspectiva de lo dominante, desde el ojo del dominador, seleccionando, escogiendo y apartando lo que se consideraba conveniente. Esa incorporación de lo popular a lo nacional, entonces, es una incorporación "pasteurizada", resemantizada en función de los ojos de quien ve, de quien dice, de quien escribe, de quien tiene la sartén por el mango. Quien tiene el poder político tiene los medios para fijar en la memoria aquello que considera pertinente, rescatable de lo que le rodea. 
Los sectores populares, que en el siglo XIX ya habían visto bastante constreñidas sus prácticas por la avalancha de manufacturas inglesas, vieron también, entonces, cómo los giros de su lenguaje, la arquitectura de sus casas, los bailes de sus celebraciones eran espulgados, seleccionados y modificados para que, una vez que asumían una forma aceptable, civilizada, fueran entendidos como parte de la naciente nacionalidad. Pero eso no sucedió con todas las expresiones de las culturas populares. Otras eran totalmente marginadas o ignoradas, cuando no perseguidas hasta el exterminio.

El proceso de construcción de la identidad cultural de la nación implicó una dinámica de homogenización cultural que pretendió que todos los habitantes del estado nacional debían sentir como propios los mismos valores y las mismas tradiciones; en una palabra, que debían compartir una sola cultura: la cultura nacional. Para ello, fue necesario no sólo inventar esos patrones comunes sino, también, borrar aquellos que no estaban acordes con el modelo a construir.

Esta situación alcanzó niveles escalofriantes en países como Guatemala, por ejemplo, en donde más de la mitad de la población no se identificaba (ni se identifica aún ahora, 100 años después de iniciado ese proceso) con la cultura nacional dominante. En ese país, más de la mitad de la población es indígena y tiene una serie de valores que no coinciden con los de la minoría dominante ladina. Pero eso no sucedió solamente en ese país: en Costa Rica la cultura nacional se concibió a sí misma como blanca, por ejemplo, borrando de un plumazo la incuestionable presencia del negro en el período colonial: lo hizo invisible.

Quiero decir con todo esto que nuestra cultura nacional es un producto en el cual están presentes una serie de mitos, transfiguraciones e inventos que sólo hasta nuestros días empezamos a desentrañar de forma sistemática. En ella se encuentran integrados muchos elementos provenientes de las culturas populares más disímiles y de distintos momentos históricos. A través suyo, esta cultura nacional, hegemónica, obtiene consenso y legitimidad ante nuestros ojos, haciéndonos partícipes. Hacer evidente esta situación es importante y necesario en la medida en que la dinámica de la cultura que se encuentra encerrada en las artificiales fronteras de los estados nacionales, siempre ha sido variada y múltiple, pero ha estado soterrada (para usar un término que utilizaron los sandinistas cuando se refirieron a la forma como había sobrevivido la cultura ligada a la gesta de Sandino en su país en el tiempo de Somoza).

Hoy, gracias a una serie de procesos económicos, sociales y culturales nuevos, nos damos cuenta de forma clara de esta situación. Las razones que nos ayudan a darnos cuenta de ella son: 
1) el debilitamiento de los estados nacionales en el marco de la transnacionalización y globalización, principalmente de la economía y las finanzas, que le restringen cada vez más sus posibilidades de autonomía, con lo que merma sus capacidades de establecer legitimación hacia el interior del estado nación.

2) los fenómenos de la globalización cultural, entre los que se incluyen las implicaciones de las migraciones masivas en todas partes del mundo, los efectos de los medios de comunicación y las industrias culturales.

Todas estas circunstancias, en las que nuevos fenómenos y procesos se hacen presentes, han redefinido el papel de las culturas populares en la conformación de las identidades colectivas, y también las han redefinido a ellas mismas. En todo este panorama existen, sin embargo, fenómenos aparentemente contradictorios, que responden a corrientes y tendencias distintas que coexisten en el mundo contemporáneo.

Por un lado tenemos todas las manifestaciones resultantes del poderoso afianzamiento del capitalismo y, por ende, de la sociedad de consumo en la vida contemporánea de nuestro mundo. El avance y universalización del mercado como elemento catalizador y perfilador de toda la vida social, ha incidido de manera importante en las culturas y las identidades populares. En este sentido, es evidente que, casi sin exclusión, en el mundo contemporáneo prácticamente no existe población que no se encuentre bajo el influjo de una u otra manifestación de la cultura de masas asociada a la sociedad de consumo.

Esta situación ha llevado a procesos disímiles en función de las circunstancias concretas: en algunos casos, tal como han temido muchas veces los cultores populares, ha llevado a la desaparición no sólo de manifestaciones culturales puntuales, sino de modos de vida y visiones mundo determinada, que se han diluido o perdido su diferencia específica en el marco del modo de vida de la sociedad occidental en su forma actual de existencia. Estas circunstancias son, sin embargo, el corolario de largos procesos de transformación que se han venido produciendo desde hace mucho tiempo, como parte de la gradual expansión del capitalismo y de la cultura occidental a él asociada, pero que han conocido un aceleramiento impetuoso en los últimos decenios.

Esta conversión ha gestado nuevas expresiones populares que van desde las de carácter lumpen hasta las más integradas, pero no por ello menos marginadas, que son expresión de sectores sociales como las de los trabajadores urbanos de los cinturones industriales o las de los trabajadores informales, entre otras. 
Estas identidades se perfilan, en muy buena medida, atenidas a los patrones que dicta la sociedad de consumo de masas, la cual orienta en valores, rasgos grupales, modelos de comportamiento, referentes icónicos, etc. Los canales a través de las cuales se reproduce este tipo de cultura son, en primer lugar, los medios de comunicación de masas, especialmente la televisión. Cuando hablamos de la televisión deben hacerse algunas precisiones: no se trata de todo lo que ese medio transmite, sino especialmente de aquellos programas que muchas veces han sido calificados como light o chatarra. Los shows en vivo, en los cuales participa un público de muchachos y muchachas adolescentes entusiastas, son alimentados por estos sectores populares quienes, además, se han ido convirtiendo en los principales consumidores de la comida también llamada chatarra, los Burguer King, los Mac Donalds, los Taco Bell, etc. que les ofrecen comida a bajo precio junto a espacios seguros de diversión a sus hijos en sus playplace.

Estos grupos sociales, que perfilan hoy su cultura (popular) influenciados de manera esencial por la cultura occidental en su versión actual de sociedad de consumo masivo, son sobre todo urbanos (aunque no solamente), lo cual no es poco decir porque estamos en un contexto en el cual vivimos un aceleradísimo proceso de urbanización a nivel planetario. En estos momentos, en
América Central, un territorio que se ha caracterizado por tener una economía principalmente asociada a la agricultura, casi un 50\% de la población se encuentra viviendo en ciudades. En el caso cercano que nos toca, Costa Rica, en un solo gran conglomerado urbano llamado Gran Área Metropolitana, ubicado en un pequeño valle intermontano, vive atosigado, sofocado y enojado el $48.3 \%$ de la población del país. Según datos del Programa de las Naciones Unidas para el Desarrollo, en treinta años más del 65\% de la población mundial vivirá en las ciudades.

Estas culturas populares urbanas a las que nos estamos refiriendo son, por lo tanto, las más extendidas y las que prevalecerán en el futuro. La ciudad, con todas sus contradicciones, es su nicho, ella les ofrece sus posibilidades y sus límites. Seguramente que la mayoría de los lectores se encuentra integrada, de una u otra forma, a este tipo de cultura. En ella establecemos variantes de prestigio, nichos de consumo que nos diferencian, culturalmente, entre nosotros. He apuntado a la palabra clave: consumo. Porque es a través del consumo como se establecen las tan preciadas y buscadas diferencias culturales de nuestros días, las cuales nos proporcionan afinidad con ciertos grupos y distancia con otros. Dependiendo de los grupos de edad, las marcas comerciales de los artículos que consumimos constituyen, hoy por hoy, un signo cultural de diferencia, así como

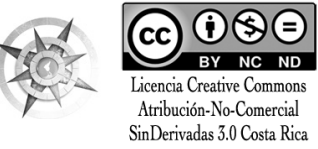


el no usar marcas es otro. Se trata de múltiples segmentos de mercado que establece diferencias estamentarias que, a la vez, encuentra referentes a escala planetaria: una adolescente costarricense perfectamente encontrará tema de conversación y afinidades con otra de Singapur por su culto a Madonna o Britney Spears, su apego a la estética de los videoclips de MTV y su gusto por ciertos tipos de comida a la que ya he hecho mención anteriormente.

Quiere decir todo esto que, cuando hablamos de identidades asociadas a las culturas populares en nuestros días es necesario tomar en cuenta todas estas transformaciones dinámicas que le están dando, lo queramos o no, el perfil a la época que nos tocó vivir. Pero, así como -en mi opinión- se debe estar alerta en relación con estos cambios culturales vertiginosos que nos preocupan por incidir de manera no siempre deseable sobre nuestras identidades, existen otros que apuntan tendencialmente en direcciones diferentes a las señaladas arriba, aunque se encuentran, ellos también, inmersos en la dinámica general de nuestros días.

Al igual que los anteriores que hemos venido mencionando, podemos ubicarlos en el campo de las culturas populares; pero sus diferencias con ellas nos muestran, entre otras cosas, que hablar de lo popular no debe remitirnos nece- sariamente a pensar en procesos de afirmación cultural de corte positivo. Estas tendencias debemos asociarlas, en buena medida, primero, al florecimiento de grupos sociales cuyas necesidades y reivindicaciones tienen un carácter grupal relativamente restringido que buscan modificar a su favor ciertos espacios de lo social que le son adversos, lo cual implica, generalmente, transformaciones en el orden de lo ideológico-cultural y, segundo, al fenómeno que ya apunté con anterioridad de debilitamiento de los estados-nación, la cual permite el resurgimiento de la variedad y pluralidad cultural que se encontró, por años, sujeta a la égida hegemónica de la cultura y la identidad nacional construida desde lo oficial.

En lo que respecta a los nuevos movimientos sociales, estos apuestan por una nueva cultura, que implica relaciones de mayor horizontalidad, de mayor participación popular en la toma de decisiones políticas y de respeto a la diversidad. La amplia variedad de grupos que existen, con ámbitos específicos de preocupación y de acción, nos dan una muestra no solamente de su riqueza sino, también, del enorme potencial que encierra la sociedad mundial contemporánea, que no se deja atrapar en los cánones de la cultura que circula por los canales globales dominantes. 
Los nuevos movimientos sociales han ido adquiriendo cada vez un mayor protagonismo en la escena mundial especialmente desde que tomando conciencia de su magnitud y fuerza, se han sabido aglutinar para tratar de incidir sobre procesos que se encuentran en marcha, y que deben entenderse como parte de la globalización. En este sentido, debe mencionarse las multitudinarias concentraciones de protesta contra la las políticas del Banco Mundial, de la Organización Mundial del Comercio, contra el ALCA, en América Latina, y las hasta ahora dos ediciones del Foro Social Mundial que se han llevado a cabo en Porto Alegre, Brasil.

Bajo el lema de "Otro mundo es posible", en el Foro Social Mundial se traza un mundo heterogéneo que, de alguna forma, es expresión de lo que la cultura popular es: múltiple, variada, expresión de la vida y de sus necesidades. Todos aquellos que, en cualquier rincón del mundo, incluyendo a Centroamérica y Costa Rica, estén interesados en lo popular, en la participación democrática, en la cultura rica y viva que emana de la práctica de los hombres y mujeres que no se sienten representados en los grandes foros de las naciones desarrolladas y de los grandes organismos mundiales, como el Fondo Monetario Internacional, el Banco Mundial, el Banco Interamericano de Desarrollo la Organización Mundial del Comercio, deben estar pendientes, vincularse de alguna forma y acuerpar este inmenso esfuerzo colectivo. En ese sentido, propongo que es necesario identificar las vías para entrar en contacto con este movimiento, con una vinculación real y efectiva, haciéndonos partícipes de estos esfuerzos. La cultura de los sectores subalternos, aquella que no alcanza los medios de comunicación de masas o que si llega a ellos es a través del filtro del mercado, tuvo un lugar de resistencia global en el Foro Social Mundial que en junio del 2004 tuvo su siguiente cita en Brasil.

Anteriormente hice mención del hecho que el debilitamiento de los estados-nación contemporáneos ha traído consigo el resurgimiento de expresiones culturales que se mantuvieron sujetas a la cultura nacional hegemónica durante decenios. No es casual que el tema del multiculturalismo esté hoy sobre el tapete. Esto se debe a varios factores que concomitantemente y con distinta profundidad, inciden sobre la marcha de los acontecimientos. Uno de ellos es, efectivamente, el de las posibilidades que han abierto los medios de comunicación de masas de observar otras formas de ser, lo que ha contribuido a la toma de conciencia de nuestra multifacética existencia como humanos. Otro factor importante es el del surgimiento de importantes movimientos étnicos, tanto en aquellos territorios en donde, hasta hace pocos años, poderosos estados ejercían un férreo control sobre nacionalidades 
consideradas minoritarias, como es el caso de la antigua Unión de Repúblicas Socialistas Soviéticas y de Yugoslavia, sobre cuyos restos han surgido viejas reivindicaciones territoriales y ancestrales enconos, como en naciones pobres en donde ciertos grupos han encontrado en la cultura tradicional un poderos elemento aglutinador y movilizador.

Es el caso de los vigorosísimos movimientos islámicos que hoy por hoy han alcanzado un protagonismo inusitado en la palestra mundial, generando incluso teorías sobre el choque de civilizaciones, como las de Samuel Hunttington, que reedita, en otro nivel y momento histórico, aquellas viejas posiciones que en América Latina hicieron carrera en el siglo XIX, las cuales tachaban de barbarie a todo aquello que fuera diferente a la cultura occidental moderna de aquellos tiempos.

Por último, quiero mencionar otro factor que contribuye al resurgimiento de todos estos movimientos reivindicatorios de las identidades étnicas y populares: el de la cada vez más limitadas posibilidades de los estados nacionales de tomar decisiones y orientar sus desarrollo en función de sus propias condiciones y necesidades, dada la prevalencia de políticas y orientaciones que surgen desde los centros de poder mundial, aquellos que no han sido electos por nadie y que responden a los intereses de los más poderosos de la Tierra. Los organismos financieros internacionales restringen, pues, cada vez más, esa autonomía de los estados nacionales.

A causa de todos esos factores y otros más que no he mencionado ahora, como es el del progresivo fenómeno del turismo internacional, la multiculturalidad aparece en nuestros días como un fenómeno crecientemente concientizado en el que las distintas expresiones populares adquieren cada vez mayor protagonismo, el cual tiene una dimensión política de primer orden.

En términos generales, lo popular se encuentra hoy por hoy inmerso en una dinámica que le obliga a ser beligerante en relación con las fuerzas que dominan la producción de valores culturales que pretenden otorgarle sentido e identidad a vastos sectores de la población mundial.

Estos grandes productores de sentidos e identidades no son más ni menos que aquellos que poseen los medios de producción audiovisual, que en un $87 \%$ se encuentran concentrados en los Estados Unidos de América. Su producción constituye el segundo gran renglón de las exportaciones de ese país, siendo superado solamente por las exportaciones de armamento. Este gigantesco aparato productor de sentidos nos ofrece una visión del mundo que se origina en múltiples y 
variados componentes de la cultura de la cual parte, incluyendo aspectos de su cultura popular. Difundida de forma masiva por todo el mundo, ésta se convierte en instrumento de los intereses económicos e ideológicos de los sectores económica y políticamente dominantes de los Estados Unidos.

Las expresiones culturales de los muchachos que integran las pandillas del Bronx de Nueva York, o de la megaciudad de Los Ángeles, en California, son recepcionadas e imitadas por millones de jóvenes en todo el mundo. Más de sesenta mil jóvenes en Honduras, ochenta mil en Guatemala y cuarenta mil en El Salvador, viven teniendo como patrón de referencia cultural esas formas de vida lumpen que surgen y se reproducen en las grandes ciudades norteamericanas. Opuestas a ellas, en nuestro pequeño territorio centroamericano, se afirman ancestrales formas culturales como las étnicas, que apuestan por un mundo en el que, como selaña el escritor libanés Amin Maalouf, se pueda escuchar, hablar, discutir y reaccionar.

Las culturas populares, entonces, están presentes en todos los órdenes de la vida contemporánea: como parapeto de resistencia o como instrumento de penetración de intereses ajenos a ella. Han pasado los tiempos en que referirse a la cultura popular era hacerlo de forma idílica, viendo en ella el núcleo de unas identidades que se entendían como vinculadas con proyectos políticos progresistas que apuntaban a la construcción de un mundo más justo. Hoy se entiende que hay que saber discernir en ellas en varios sentidos: primero, entre lo que tienen de afirmador de la autoconciencia y la autoestima de los pueblos y lo que tienen de trasnochado haciendo pervivir formas opresivas de convivencia; segundo, entre lo que es instrumentalizado a favor de intereses que le son ajenos a las grandes mayorías y lo que le sirve de instrumento de resistencia y construcción del un futuro desde sus propios intereses.

Estimables lectores: quizá concuerden conmigo en que la época que nos ha tocado vivir es una época de tránsito. Venimos de una forma de sociedad, la moderna y vamos hacia otra forma de organización social que aún no logramos vislumbrar. Se especula mucho al respecto: ¿vamos hacia un siglo dominado por el imperio norteamericano?, ¿nos dirigimos hacia una era de democracia universal basada en los valores liberales que constituyen la cúspide del devenir histórico humano?; ¿ha llegado la hora en la que el mercado transformará todo lo que toque, como el rey Midas, en mercancía?

En función de las múltiples y diversa tendencias que nos presenta la contemporaneidad podemos especular. Lo cierto es que, independientemente de cuáles sean las tendencias que se consoliden 
en el futuro, mucho dependerá del papel que la sociedad civil, en toda su fascinante diversidad, pueda cumplir. Una tarea fundamental para todos aquellos que se encuentran comprometidos con las diversas expresiones de lo popular, ya sean estás culturales o políticas, económicas o sociales, es afirmar la conciencia de que otro mundo es posible, y que está en nuestras manos imaginarlo, impulsarlo y construirlo. Desde esta perspectiva, las culturas populares, sin lugar a dudas, tendrán un papel protagónico en varios sentidos: en primer lugar, como aglutinadoras de voluntades; en segundo lugar, como inspiradoras de autoestima y seguridad en nosotros mismos y, en tercer lugar, aportando elementos para los proyectos de construcción del futuro.

Todo esto significa entender a las culturas populares como parte de un movimiento político que juega un papel beligerante en nuestros días, que toma partido y se enfrenta a las políticas neoliberales y a los organismos que las impulsan en todo el mundo. En la dinámica de este movimiento están cristalizando nuevas identidades, distintas muchas veces a las que conocimos en el pasado, pero que tendrán un papel protagónico en el perfilamiento del mundo que queremos para mañana.

Mi reflexión ha querido resaltar estos fenómenos de la vida contemporánea sobre los cuales, en mi opinión, debemos poner más atención pues se refieren, en última instancia, a lo que en buena parte somos hoy y seguiremos siendo en el futuro.

\section{Referencias bibliográficas}

Quesada Soto, A. (1986). La formación de la narrativa nacional costarricense (1890-1910). Enfoque histórico social. Costa Rica: EUCR 